\title{
LA FRAGILIDAD DE LAS DICOTOMÍAS SEXUALES Y DE GÉNERO EN EL PENSAMIENTO DE PAUL B. PRECIADO'
}

\author{
THE FRAGILTYY OF SEXUAL AND GENDER DICHOTOMIES
}

IN THE THOUGHT OF PAUL B. PRECIADO

\author{
Maria Medina-Vicent ${ }^{2}$ \\ Universitat Jaume I de Castellón
}

\section{RESUMEN}

Contra-sexualidad es un término que suscita amplios interrogantes y escasas certezas. Sin embargo, lo que se pretende en este pequeño escrito no es alcanzar verdades absolutas. Más bien al contrario, nuestro objetivo es interrogarnos y desmontar las verdades de género a través de la obra de Preciado. Este artículo se centrará en la noción de contra-sexualidad y su carácter subversivo dentro de un marco social heteronormativo. Una aproximación a dicho concepto nos permitirá utilizar el dildo para desvelar la plasticidad de los cuerpos, los sexos y los géneros, en un nuevo mundo caracterizado por la heterogeneidad de prácticas sexuales y normas descentradas.

Palabras clave: posffeminismo, contra-sexualidad, prótesis de género, tecnologías del sexo, dildo.

\section{ABSTRACT}

Contra-Sexuality is a term which opens a lot of questions but has limited certainties. Nevertheless, the aim of this essay is not to achieve absolute truths. On the contrary, the aim is to interrogate the truths of gender through the Preciado's Philosophy. This essay focus on the Contra-Sexuality term and its subversive nature in heteronormative society. An approach to this concept will allow us to use the dildo to reveal the plasticity of bodies, sexes and genders, in a new world with sexual practices diversity.

Keywords: Postfeminism, Contra-Sexuality, Gender Prosthesis, Sex Tecnologies, Dildo.

\footnotetext{
1 Este estudio se inscribe en el Proyecto de Investigación Científica y Desarrollo Tecnológico titulado «Ética de la democracia: crisis de la política y nuevas formas de participación de la sociedad civil» (P1.1B2013-24), financiado por el Plan de Promoción a la Investigación de la Universitat Jaume I.

2 Maria Medina-Vicent es Personal Investigador en Formación FPI-UJI (PREDOC/2013/16 - n ORCID: orcid.org/0000-00022716-6786) en el Departamento de Filosofía y Sociología de la Universitat Jaume I (Castelló de la Plana). Participa en el grupo de investigación «Filosofía política y ética empresarial» de la misma universidad. Sus líneas centrales de investigación son la filosofía feminista, los Critical Management Studies y la ética empresarial dialógica. Contacto: medinam@uji.es
} 


\section{Introducción}

El feminismo es transgresión, ya que socava los presupuestos universales y las estructuras sociales que se nos muestran como hechos naturales no susceptibles de transformación. Sin embargo, existen diferentes caminos para transgredir realidades y significados desde la teoría feminista. Partiendo de estas afirmaciones, nos adentraremos en los conceptos principales de la obra filosófica de Paul B. Preciado 3 (antes Beatriz Preciado), una figura que transgrede nuestra manera de entender el feminismo y los géneros, así como el modo generalizado de concebir el mundo.

El postfeminismo en el que se inscribe su trabajo se caracteriza por la descentralización del sujeto político del feminismo (Gill y Scharff, 2011; Gill, 2014), pasando de una noción cerrada de mujer a una incorporación de los grupos sociales históricamente excluidos en las reclamaciones feministas: lesbianas, gays, transexuales, transgénero, etc. Así, se incorporan las diferencias culturales, sexuales y políticas para desmitificar el ideal heterosexual y eurocéntrico de mujer que ha predominado en la lucha política feminista por la igualdad desde el siglo XVIII. Con el surgimiento de la teoría queer, se cuestiona pues el carácter natural y universal de la condición femenina a través de la explosión de toda una heterogeneidad de corrientes, propuestas y trabajos que dejan atrás el tradicional debate entre feminismo de la igualdad y de la diferencia, abriendo paso a un abanico inacabable de cuestiones a abordar (Lumby, 2014).

En el año 2002 Preciado publicó Manifiesto contra-sexual (Opera Prima, Madrid), considerada hoy en día una obra clave en el desarrollo de la teoría y el movimiento queer. Partiremos de esta obra para tratar el concepto de "contra-sexualidad», que contiene la intención de superar una noción natural de sexo, sexualidad y cuerpo. La contra-sexualidad es entre otras cosas «una teoría del cuerpo que se sitúa fuera de las oposiciones hombre/ mujer, masculino/femenino, heterosexualidad/homosexualidad» (Preciado, 2002: 19), va más allá de las nociones dicotómicas del ser mujer o ser hombre. Es en este sentido en el que Preciado transgrede no solamente la forma de entender el género y el cuerpo biológico, sino la forma normalizada y generalizada de concebir las relaciones amorosas, sexuales y de poder en las sociedades occidentales actuales. Con la voluntad de des-naturalizar

3 Beatriz Preciado ha cambiado recientemente su nombre por el de Paul B. Preciado, mostrando una vez más, la complejidad de su pensamiento y la imbricación de su filosofía con su vida práctica. Dicha acción puede entenderse como uno más de sus intentos por desestabilizar las fuerzas de dominación de los cuerpos, sexos y géneros a través de su propia lucha. Así, su decisión de habitar la masculinidad responde a razones vitalmente políticas, como se desprende de sus propias palabras en una entrevista realizada a mediados del año 2015: «Me cuesta pensar por qué alguien elegiría un solo género toda la vida. No veo mi situación como excepcional; lo excepcional es la inmovilidad de género en el resto de la gente. Estoy cambiando ahora, pero tal vez al final de mi vida quiera cambiar a otra cosa» (Curia, 2015).

Dossiers Feministes, 21, 2016, 5-21 - ISSN: $1139-1219$ - DOI: http://dx.doi.org/10.6035/Dossiers.2016.21.1 
y des-mitificar las nociones tradicionales de sexo y de género, la contra-sexualidad «estudia los instrumentos y los aparatos sexuales $y$, por lo tanto, las relaciones de sexo y de género que se establecen entre el cuerpo y la máquina» (Preciado, 2002: 21). Así, se conceptualiza el sexo como tecnología, y se conjuga con la idea de "prótesis de género», que resulta de vital importancia para repensar los procesos de asignación de sexo, género y sexualidad en la sociedad occidental, ya que nos permite concebir cualquier cosa/objeto como un potencial dildo.

En definitiva, este artículo es un ejercicio egoísta iniciado por el interés personal y académico que nos despierta el pensamiento de Preciado, una filosofía que nos empuja a transgredir nuestros propios límites como investigadoras, ciudadanas y mujeres. Por esta razón, a lo largo de estas páginas realizaremos un tímido acercamiento al concepto de contra-sexualidad, subrayando el papel de la prótesis de género, el sexo como tecnología y el uso del dildo para subvertir las prácticas sexuales heteronormativas que vertebran la lógica de género actual.

\section{De la contra-sexualidad y la subversión de la heteronormatividad}

Los planteamientos filosóficos de Preciado tienen la capacidad de tornar volátil y difuso todo aquello que como seres humanos inmersos en una cultura concreta, creíamos irrefutable. La aproximación a su trabajo es siempre dolorosa, por lo que de reveladora tiene para el modo convencional de percibir el mundo. De este modo, nos atrae y repele cuál objeto de deseo por el que sentimos curiosidad y que al mismo tiempo nos aterra por su fuerza irrefrenable.

En este sentido, partimos del concepto de contra-sexualidad, término clave que vertebra la obra de Preciado ya citada. El prefijo «contra» nos pone sobre aviso, ya que torna explícita la intención central del escrito, que es, desmontar los pilares de género sobre los que se sustenta el mundo y también nuestra percepción del mismo. Intentar comprender la obra de Preciado nos va a poner en una situación delicada, nos va a obligar a luchar contra nosotros/as mismos/as, contra todas las convenciones sexuales que nos han sido enseñadas y hemos incorporado casi sin pestañear, amoldándonos a una u otra identidad de género. Así, una vez reconocido el carácter transgresor de la obra de Preciado, cabe destacar que el sexo se convierte en el objeto de análisis principal, pero no a partir de la diferencia sexual o el género, sino a través del concepto de dildo que trataremos más adelante. Para comenzar, nos vemos en la necesidad de exponer la definición de contra-sexualidad que Preciado establece: 
La contra-sexualidad no es la creación de una nueva naturaleza, sino más bien el fin de la Naturaleza como orden que legitima la sujeción de unos cuerpos a otros. La contra-sexualidad es. En primer lugar: un análisis crítico de la diferencia de género y de sexo, producto del contrato social heterocentrado, cuyas performatividades normativas han sido inscritas en los cuerpos como verdades biológicas. En segundo lugar: la contra-sexualidad apunta a sustituir este contrato social que denominamos Naturaleza por un contrato contra-sexual. En el marco del contrato contra-sexual, los cuerpos se reconocen a sí mismos no como hombres o mujeres, sino como cuerpos parlantes. Se reconocen a sí mismos la posibilidad de acceder a todas las prácticas significantes, así como a todas las posiciones de enunciación, en tanto sujetos, que la historia ha determinado como masculinas, femeninas, perversas. Por consiguiente, renuncian no sólo a una identidad sexual cerrada y determinada naturalmente, sino también a los beneficios que podrían obtener de una naturalización de los efectos sociales, económicos y jurídicos de sus prácticas significantes (Preciado, 2002: 18-19).

A partir de dicha definición, se pueden extraer dos ideas principales que nos servirán de piedras angulares en el recorrido que acabamos de emprender. En primer lugar, la idea de que no solamente el género es construido cultural y socialmente, sino que también los cuerpos biológicos lo son. En segundo lugar y consecuentemente, que las prácticas heterosexuales consideradas «naturales» no son más que el fruto de un contrato artificial, cuya artificialidad intenta hacer patente Preciado mediante la propuesta de otro contrato, el contra-sexual.

En primer lugar, las normas de la sociedad heterosexual han sido incorporadas por las personas a partir de su reiteración constante en forma de enunciados de género, que sirven para construir y legitimar las identidades de género tradicionales, es decir, masculino y femenino. Así pues, el contrato normativo heterosexual se centra en ligar la sexualidad con la naturaleza, o más bien, la sexualidad heterosexual con una supuesta naturaleza biológica que diferencia entre sexo femenino y masculino. Se liga la identidad genérica (mujeres/ hombres), con ciertas prácticas sexuales (heterosexualidad), como si se fundamentasen en un principio cero o en una naturaleza primigenia que las reconoce como única vía posible para relacionarse sexualmente entre humanos. Esto quiere decir, las prácticas sexuales heterosexuales se dibujan como aquellas que son naturales, todo aquel placer que no encaje en dicha descripción será castigado y tildado de enfermedad. Esta operación semántica se realiza en gran medida a partir de la fragmentación de los cuerpos y sobre todo, de la sexualización de los órganos reproductores, el pene y la vagina, como exclusivos centros del placer del cuerpo humano. En este aspecto, se erotizan las únicas partes del cuerpo que van a servir para la reproducción de la especie y del sistema del capital. Cuán conveniente resulta esta operación para el mantenimiento del status quo es algo que nos preguntamos desde la posición de Preciado: 
El sistema heterosexual es un aparato social de producción de feminidad y masculinidad que opera por división y fragmentación del cuerpo: recorta órganos y genera zonas de alta intensidad sensitiva y motriz (visual, táctil, olfativa...) que después identifica como centros naturales y anatómicos de la diferencia sexual (Preciado, 2002: 22).

Como hemos visto, a cada género, con su correspondiente cuerpo masculino o femenino, se le asocian un tipo de prácticas sexuales, se le asignan una serie de zonas erógenas y placeres considerados naturales. El sistema heteronormativo es el encargado de edificar y otorgar significados a dichas prácticas, asociándolas a los cuerpos correspondientes. Sin embargo, la teoría contra-sexual permite ampliar estas nociones, desligando las identidades de género de las prácticas sexuales concretas, al mismo tiempo que se distorsionan las identidades de género. Consecuentemente, con el concepto de contrasexualidad «Preciado propone revocar el contrato que liga la sexualidad con la naturaleza biológica de un individuo, contrato vigente en una sociedad heterocentrada» (Vargas-Pardo, 2008: 124), y lo revoca desde la citación de otras prácticas sexuales que no sirvan solo a la reproducción humana, que no fragmenten el cuerpo y que sí ofrezcan una visión del placer sexual transgresora, considerando cualquier parte del cuerpo como posible portadora y generadora de placer. Para explosionar un conjunto infinito de posibilidades de contra atacar la sexualidad normativa Preciado se nutre de las obras de Monique Wittig, Michel Foucault, Judith Butler, Jaques Derrida y Donna Haraway.

\section{Performatividad: los enunciados de género y su carácter prescriptivo}

La heterosexualidad se reinscribe en los cuerpos femeninos y masculinos de forma constante, respondiendo a la norma central de una sociedad heteronormativa. En este sentido, los trabajos de Judith Butler, permiten a Preciado reconocer desde la idea de performatividad (Butler, 2002), los mecanismos de normalización de género que son puestos en práctica desde las estructuras estatales, educativas, médicas, etc., entre otras. En este sentido, Butler entiende que las identidades de género son construidas a través de los discursos institucionalizados sobre esas mismas identidades. Es decir, son construcciones culturales realizadas a través del lenguaje, aunque no de forma exclusiva, que se dan a través de un proceso discursivo en el que la sanción ejerce un papel central. Y es que la sanción social de toda práctica que se escape de lo heteronormativo refuerza las identidades de género supuestamente naturales. Toda aquella conducta, apariencia, etc. que se escape de la norma será sancionada en un intento por reconducirla hacia lo establecido, legitimando así su existencia como desviación. Por tanto, el concepto de performatividad empleado por Butler, sirve para discernir el poder que tiene 
el discurso institucional de género sobre los cuerpos y sobre la naturalización del proceso político de creación de las identidades de género:

De esta forma, el género y el sexo son actuaciones, actos performativos, que son modalidades del discurso autoritario; tal performatividad alude en el mismo sentido al poder del discurso para realizar (producir) aquello que enuncia, y por lo tanto permite reflexionar acerca de cómo el poder hegemónico heterocentrado actúa como discurso creador de realidades socioculturales. En este sentido, y en la línea foucaultiana, puede entenderse la performatividad del lenguaje como una tecnología; como un dispositivo de poder social y político (Duque, 2010: 87).

Como se desprende de la anterior cita de Duque, al nacer, las personas son incorporadas como sujetos sociales dentro de un sistema heteronormativo. A través de la constante identificación de sus cuerpos y sus prácticas sexuales con la norma heterosexual establecida, esas mismas personas van a encargarse de reproducir y legitimar la heteronormatividad. En dicho proceso, los enunciados de género (es niño o es niña) ejercerán un papel central, en tanto enunciados que aparentemente describen una realidad objetiva, pero que son actos performativos que imponen y reproducen una convención social, una verdad política.

En este caso, la verdad política recae en el reconocimiento de dos géneros supuestamente naturales, el masculino y el femenino, que se asociarían a su vez con dos cuerpos biológicos concretos y una práctica sexual concreta, esto es, la heterosexual. Lo que se acaba de exponer a través de la teoría de Butler, conduce a la redefinición de la noción de género, que afirma que la identidad de género no sería algo sustancial, sino el efecto performativo de la invocación de una serie de convenciones de feminidad y masculinidad. Sin embargo, desde el punto de vista de Preciado, Butler se olvida demasiado pronto del cuerpo, de la materialidad que sostiene dichas identidades supuestamente performativas (Preciado, 2002: 75). Según Preciado, el cuerpo también es construido como arquitectura política, ya que está fragmentado en partes dicotómicas: permitido/no permitido, activo/ pasivo, placer/dolor, masculino/femenino:

El género no es simplemente performativo (es decir, un efecto de las prácticas culturales lingüístico-discursivas) como habría querido Judith Butler. El género es ante todo prostético, es decir, no se da sino en la materialidad de los cuerpos. Es puramente construido y al mismo tiempo enteramente orgánico. Escapa a las falsas dicotomías metafísicas entre el cuerpo y el alma, la forma y la materia (Preciado, 2002: 25). 
Una de las transgresiones de Preciado estriba en no solamente reconocer el carácter constructivista de los géneros, sino subrayar que la materialidad corpórea sobre la que se edifican dichos géneros también es construida. Para llegar a comprender estas afirmaciones, debemos introducirnos en la comprensión del sexo como tecnología. Si bien es cierto, la propuesta de Preciado pretende reformular las bases sociales, apostando por una sociedad en que se deconstruyan las concepciones naturales del sexo y el género. $O$ lo que es lo mismo, deconstruir las prácticas sexuales que se han convertido en la norma, para abrir paso a un abanico heterogéneo de posibilidades al tiempo que se desestabilizan las nociones «naturales» del género y la sexualidad. Debemos partir del reconocimiento de que según esta perspectiva, no existen cuerpos terminados, no existen cuerpos finitos. El sexo es tecnología que se reinventa a través de las prótesis.

\section{De la tecnología del sexo o del poder político del bisturí}

El debate esencialismo - constructivismo ha vertebrado el feminismo desde hace décadas, sin embargo, para Preciado dicho ir y venir resulta estéril y contraproducente. Desde su postura, ambas partes del debate tornan esenciales los cuerpos como si se tratasen de un punto cero desde el que se va a construir después el género. Es decir, tanto los feminismos de corte esencialista como los de corte constructivista parten de un cuerpo físico supuestamente natural al que asignan capacidades y valores, de forma esencial en el primer caso, de forma cultural en el segundo. Pero en ningún momento renuncian a ese cuerpo biológico aparentemente imperturbable. Así, «los dos modelos dependen de un presupuesto moderno: la creencia según la cual el cuerpo entraña un grado cero o verdad última, una materia biológica (...) dada» (Preciado, 2002: 126). Sin embargo, los avances en la transformación de los órganos sexuales y los cuerpos mediante intervenciones quirúrgicas, que Preciado menciona y por las cuales hace un recorrido, muestran que no es solamente el género lo que se construye cultural y normativamente hablando, sino el cuerpo orgánico el que es construido y modificado a golpe de bisturí y fármacos.

Para ilustrar la artificialidad de los géneros y los sexos, Preciado reflexiona sobre la asignación de sexo de los bebés intersexuales y los casos de personas transexuales. Al nacer, en la camilla del hospital da comienzo el proceso de asignación sexual que será institucionalizado a lo largo de la vida del sujeto a través de instituciones como la familia, la escuela y el Estado. En su trabajo, Preciado nos hace reflexionar sobre la escasa cientificidad de los procesos en que un bebé es reconocido como niño o niña, ya que la elección es ante todo visual. 
En los casos de intersexualidad, donde los órganos no se corresponden con la norma, ya sea por tamaño, forma u otras cuestiones, la determinación de si dicho bebé será una mujer o un hombre, resulta igualmente gratuita. Un ejemplo revelador que expone Preciado, es el caso de bebés cuyo pene es más pequeño de lo «normal», los llamados micropenes. En muchas ocasiones se decide convertir dicho pene en una vagina para ahorrar trastornos futuros a la persona en cuestión. Como vemos, la identidad sexual se decide de forma visual, y es transformada tecnológicamente. Así, los bebés intersexuales ponen en funcionamiento dos tecnologías diferenciadas, una genética esencialista y otra quirúrgica constructivista (Preciado, 2002: 113). A través de las creencias esencialistas basadas en el reconocimiento de una dualidad de cuerpos (masculino y femenino) cero, todo aquello que no entre dentro de dicha pareja, será intervenido tecnológicamente para construirse como tal.

Asignar a estos bebés un sexo y un género contiene valor prescriptivo, no descriptivo. Crear un órgano sexual determinado, poner un nombre propio... son enunciados prescriptivos que intervendrán en la formación de esa persona como mujer u hombre heterosexual. Por tanto, se esclarece un hecho y es que no existe una naturaleza sexual o genérica preexistente, sino que la misma acción de nombrarla es una operación semántica que inscribe sobre los cuerpos unos significados concretos. Es decir, existe todo un entramado de tecnologías que operan para construir sexo y género de forma natural. En relación, Preciado señala que «la arquitectura funciona como una verdadera prótesis de género que produce y fija las diferencias entre tales funciones biológicas» (Preciado, 2006: 44).

Así, los urinarios públicos perpetúan diferentes dicotomías entre las que destacan: público/privado, masculino/femenino, mear/cagar, etc. Cada género tiene asignado su espacio para cagar y mear, cada cuerpo que entra es sancionado si no se corresponde con el género oportuno. Por ejemplo, una mujer muy masculina, será sancionada visual y verbalmente, y se le pedirá que vaya al lavabo que le corresponde. En el caso de los hombres, la evidencia está más clara. Se encuentra estipulado que hay que mear de pie y cagar sentado. Las mujeres realizan ambas acciones sentadas, como signo de pasividad. Sin embargo, para los hombres «mear de pie públicamente es una de las performances constitutivas de la masculinidad heterosexual moderna» (Preciado, 2006: 44), mientras que al cagar, operación realizada a través del ano, la posición es pasiva, para evitar posibles actividades homoeróticas. Como se pude observar, el ano es convertido en un elemento pasivo, mientras el pene se convierte en una exposición pública de la masculinidad y la heterosexualidad. De este modo, «el discreto urinario no es tanto un instrumento de higiene como una tecnología de género que participa en la producción de la masculinidad en el espacio público» (Preciado, 2006: 44). Por tanto, existe todo un entramado ideológico que opera a través de diferentes 
tecnologías, como por ejemplo la arquitectura, que actúan como dispositivos de control en vistas a la legitimación de la norma heterosexual.

Para comprender la identificación del sexo como tecnología, Preciado parte de las aportaciones foucaultianas al concepto de poder. Así, Foucault «observa el conjunto de fenómenos que tuvieron lugar entre los siglos XVII y XVIII, cuando el Estado comienza a ejercer su soberanía sobre los cuerpos y despliega lo que puede denominarse una ««tecnología de la seguridad biológica» aplicada sobre el cuerpo» (Aguilera, 2010: 33), operación que es identificada con la biopolítica. Dicho concepto nos remite a las estrategias de poder que tienen por objeto ejercer un control total sobre la vida de las personas y sus cuerpos en la sociedad moderna. Así pues, la biopolítica produce la verdad, tiene carácter productivo «tanto si entendemos esta categoría desde la perspectiva de la producción de conocimiento, de producción de la verdad, por decirlo en términos foucaultianos, como si nos referimos a través de ella a la creación de bienes y servicios, tout court» (Fernández, 2009: 97). De esta suerte, a diferencia de Marx, Foucault no entiende el poder como exclusivamente emergente de las estructuras económicas, sino como un complejo entramado de relaciones sociales. En este sentido, el poder político para producir verdades se inscribe sobre los cuerpos a través de la biopolítica, que se encuentra en todos los ámbitos sociales:

Para Foucault, una técnica es un dispositivo complejo de poder y de saber que integra los instrumentos y los textos, los discursos y los regímenes del cuerpo, las leyes y las reglas para la maximización de la vida, los placeres del cuerpo y la regulación de los enunciados de verdad (Preciado, 2002: 124).

La regulación de los cuerpos bajo un sistema heteronormativo se nutre de los aparatos médicos, educativos, mediáticos, etc. para configurar aquello considerado natural. El caso de las personas adultas que desean cambiar de sexo también resulta de gran interés en esta línea. Y es que, nos muestran cómo la tecnología, la industria farmacéutica y la médica operan como mecanismos de transformación de los cuerpos, revelando que también la materialidad física de las personas es susceptible de ser modificada para encajar en el sistema heteronormativo. Sin embargo, Preciado señala que la tendencia predominante en los cambios de sexo o en la asignación de sexo a bebés intersexuales es transformar penes en vaginas. Sobre todo en los últimos casos, la tendencia es a escoger el género femenino. En su corrección para uniformizar a estos bebés hacia la norma, se ve mucho más claro cómo la verdad masculina es construida como verdad absoluta en las sociedades modernas. Es decir, el pene y la masculinidad se consideran portantes intrínsecos de lo femenino 
y no al contrario. Esto quiere decir que el pene puede devenir vagina con mayor facilidad que la operación inversa:

De hecho, las técnicas de producción de la masculinidad y de la feminidad están truncadas: la masculinidad se realiza según un modelo hermafrodita que permite el paso «natural» del pene a la vagina, mientras que la feminidad obedece a un modelo de producción del sexo irreversible, un modelo prostético, en el que un trozo de brazo o de pierna puede ser transformado en pene (Preciado, 2002: 101).

A pesar de afanarse en legitimar la creencia de la naturaleza indiscutible de dos sexos y dos géneros, la medicina moderna y ahora, la industria farmacéutica, han mostrado que no es tan simple. A través de la posibilidad de intervenir los cuerpos no normativos para normalizarlos en base a un esquema mujer/hombre; se ha demostrado que el cuerpo es plástico, es moldeable y puede estar sujeto a cambios quirúrgicos. Por tanto, afirmar que el sexo es prostético no parece ninguna locura. A pesar de que en muchos casos, estas intervenciones quirúrgicas sirven para «restituir un supuesto momento original» (Preciado, 2002: 110), la misma operación revela lo artificial de los sexos, ya que, a golpe de bisturí estos pueden ser construidos, no sin dificultad.

Como bien señala Preciado, somos incapaces de aceptar un cuerpo que se escape de la norma, que no coincida con nuestra imagen de lo que es femenino y lo que es masculino, de ahí esa necesidad de cortar y coser para devolver a ese supuesto momento original a la persona que no puede ser fácilmente recolocada en una de ambas categorías sexuales. Por tanto, «en su concepción, la heterosexualidad es una «tecnología social» y no un origen natural fundador, y por ello resulta posible invertir y derivar sus prácticas e identidad sexual» (Rodríguez, 2003: 91), transgredir dichas prácticas es una de las tareas que nos plantea Preciado. De buen grado, lo consigue en su obra Testo Yonqui (Preciado, 2008), donde experimenta con su propio cuerpo el poder político de la testosterona, medida de experimentación autocobaya que sigue desarrollando en la actualidad, transformando su cuerpo y encarnando en su propio ser el cuestionamiento de los regímenes biopolíticos.

\section{Prótesis de género: el dildo y la plasticidad de los sexos}

Del anterior apartado se desprende la tesis de Preciado sobre el carácter construido no solamente del género, sino del sexo y del cuerpo. Para ilustrar dichas afirmaciones Preciado «construye un concepto, el de «prótesis de género», que le sirve para pensar la no naturalidad del sexo y el género, su carácter artificial o construido, fruto de procesos de trans- 
formación» (Balza, 2011: 29). Tradicionalmente, se reduce el pene a único órgano capaz de producir el impulso sexual. Sin embargo, Preciado utiliza el dildo para revelar la plasticidad de los sexos, la artificialidad de los órganos sexuales y de la heteronormatividad.

Para empezar, partimos de que el uso del dildo en las relaciones lésbicas pone en juego una cuestión: si el uso del dildo sirve como sustituto del pene. Sin embargo, Preciado lleva más allá el tema, y nos muestra cómo la verdadera importancia no radica en dicha sustitución, sino en la operación de resignificación epistemológica que se lleva a cabo mediante la creación y el uso del dildo en las relaciones sexuales. Porque «el dildo es el primer indicador de la plasticidad sexual del cuerpo y de la posible modificación prostética de su contorno» (Preciado, 2002: 63), debido a que amplía los cuerpos y transforma el placer sexual, pasándolo del cuerpo a la máquina. Se trataría pues de un órgano de plástico, que en su materialidad y forma misma supera la noción de órgano sexual natural encarnada en el pene. Así pues, «la invención del dildo supone el final del pene como origen de la diferencia sexual» (Preciado, 2002: 64), el ser hombre o mujer ya no radica en una tenencia o ausencia del pene, ya que cualquiera puede apoderarse del dildo. Se rompe la norma, el pene ya no es el único portador del placer. En este sentido, Preciado plantea que «la teoría lésbica separatista que critica la utilización del dildo, por su complicidad con los signos de la dominación masculina, cree todavía en la realidad del pene como sexo» (Preciado, 2002: 68).

El dildo pone en evidencia la plasticidad de los cuerpos, e incluso de los órganos sexuales. En primer lugar, «pone en cuestión la idea según la cual el cuerpo masculino es el contexto natural de la prótesis del pene. Después, y de un modo más drástico, amenaza la suposición según la cual el cuerpo orgánico es el contexto propio de la sexualidad» (Preciado, 2002: 69-70). Así, el dildo, al imitar al pene, resituándolo en otros cuerpos y en otras zonas corporales, replantea la inmutabilidad del pene como órgano que solamente puede encontrarse en los cuerpos categorizados como masculinos. Al mismo tiempo, destierra la creencia institucionalizada de que el placer se sitúa en los cuerpos biológicos, y en zonas concretas, dando paso al replanteamiento de las relaciones heterosexuales como las únicas posibles. Por tanto, se sitúa contra la parcialización del placer en los cuerpos:

En esta línea, el dildo constituye su operador conceptual para demostrar el efecto prostético de la tecnología en la constitución del cuerpo, originando una deconstrucción de la naturalización de las prácticas sexuales. Para la autora, el dildo antecede al pene y así nos conduce a concebir la transformación tecnológica de los cuerpos, por ende su producción no natural (Martínez, 2012: 192). 
Para comprender mejor el papel del dildo en la filosofía de Preciado, debemos atender a dos hechos: el nacimiento de las prótesis médicas y la invención del dildo vibrador para el tratamiento de la histeria en las mujeres. En primer lugar, después de la Primera Guerra Mundial, la vuelta a casa de soldados mutilados da lugar a un gran interés en la fabricación y estudio de prótesis, pensadas no solamente como una sustitución inanimada del miembro perdido, sino como prótesis que sirviesen para que la persona pudiera seguir siendo productiva para la sociedad. Sin embargo, lo que nos interesa de la prótesis no es la sustitución de un miembro, sino la operación de resignificar los cuerpos que lleva a cabo, «porque la prótesis no reemplaza solamente a un órgano ausente; es también la modificación y el desarrollo de un órgano vivo con la ayuda de un suplemento tecnológico» (Preciado, 2002: 132). La prótesis modifica el cuerpo de tal modo que lo convierte en algo totalmente distinto, lo que Haraway denominaría un cyborg (1991), y que Preciado define como post-humano.

Por otro lado, el dildo vibrador nace como tratamiento a una enfermedad categorizada exclusivamente como femenina: la histeria. El tratamiento de dicha enfermedad consistía en una titilación del clítoris o en el uso del dildo vibrador, que culminaba en una crisis nerviosa de la mujer, un orgasmo. En la enfermedad, el dildo vibrador sirve para la cura de la mujer, y no existe peligro ya que «es la máquina la que tiene un orgasmo. No hay, por tanto, ni responsabilidad sexual, ni verdadero sujeto del placer» (Preciado, 2002: 93). Así pues, el dildo está relacionado con tres tecnologías distintas, una primera de represión del placer como puede ser el cinturón antimasturbatorio; una segunda de producción del placer como la máquina vibradora; y una tercera, relacionada con los implantes prostéticos que emergen especialmente después de la Primera Guerra Mundial. Teniendo en cuenta estas tres ideas, más que como una imitación del pene, el dildo vibrador se ha convertido en la mano lesbiana. Esto nos permite ver cómo la máquina se vuelve protagonista del placer. En este sentido, la imagen titulada Molly (1973) de Michel A. Rosen, nos permite concebir visualmente de lo que estamos hablando, Preciado la incorpora en su manifiesto y Cano nos explica lo siguiente:

Otro ejemplo de descentralización del placer, de la genitalia y de la penetración es Molly (1973) de Michel A. Rosen. Una mujer desnuda, rapada, muy maquillada, que ríe a mandíbula batiente estimula con un vibrador al dildo que lleva puesto con arnés. Este retruécano muestra la posibilidad de la sexualidad posthumana. Ya no importa la identidad o la orientación sexual. El placer es producido y se siente fuera del cuerpo. Puede entenderse este vibrador como la extensión mecánica de la mano y el dildo como la extensión no carnal del clítoris. «Tecnología» y placer. Molly es una imagen sintetizadora de la contra-sexualidad formulada por Beatriz Preciado (Cano, 2003: 156). 
En resumen, a través del dildo, Preciado nos permite ver cómo las prótesis de plástico permiten transformar el sexo de forma constante: «(...) el sexo y el género deberían considerarse como formas de incorporación prostética que se hacen pasar por naturales, pero que, pese a su resistencia anatómico-política, están sujetos a procesos constantes de transformación y de cambio» (Preciado, 2002: 134). Los cuerpos no permanecen estables, los límites de los cuerpos están por descubrir, si es que existen. Las prácticas heterosexuales no son las únicas posibles en las relaciones humanas. Así, se transforma la sexualidad, resituando los centros de placer.

\section{Por un nuevo contrato: propuesta de prácticas contra-sexuales}

El Manifiesto contra-sexual no solamente reflexiona sobre los límites de los cuerpos y la sexualidad, sino que explicita los principios que deben regir a esta nueva sociedad contra-sexual. La intención central recae en borrar las denominaciones masculino/femenino y sus asociados biológicos hombre/mujer. Así, lo más importante a la hora de subvertir estas dicotomías normativas es la incorporación de políticas contra-sexuales que parodien aquellos fenómenos sexuales considerados naturales, mostrando su artificialidad. En este sentido, Preciado «propone un conjunto de políticas regidas por la noción de principio autocobaya como modo de producción de saber y transformación política» (López Pascual, 2012: 11), o lo que es lo mismo, poner en práctica en el cuerpo de cada cual la nueva lógica contra-sexual.

Entre otras cosas, Preciado propone la simulación del orgasmo y la resexualización del ano como dos de las bases sobre las que se deben sustentar las prácticas contra-sexuales (Preciado, 2002: 29-38). En el epílogo de la obra El deseo sexual de Guy Hocquenghem concibe el ano como fuente de lucha política, ya que «el ano no tiene sexo, ni género, como la mano, escapa a la retórica de la diferencia sexual» (Preciado, 2009: 171). Además, a modo de manual, explicita los pasos, materiales y demás componentes necesarios para llevar a cabo de buen grado las prácticas contra-sexuales. Con sus propuestas concretas evidencia que su reflexión teórica está intrínsecamente conectada a su compromiso político.

Un ejemplo que ya hemos citado es su obra del año 2008, Testo yonqui, donde Preciado experimenta con su propio cuerpo las idas y venidas de los tratamientos con testosterona, así, interconecta la narración de sus vivencias como sujeto de la experimentación con el trabajo de grandes figuras del pensamiento filosófico contemporáneo. Se trata pues de crear una sociedad donde se desnaturalicen las relaciones sexuales y genéricas construidas como naturales. El habitante de esta sociedad es el ser posthumano, formado por organismo y tecnología, sujetos con prótesis tecnológicas, protagonistas de las prácticas contra-sexuales: 
«los sujetos contra-sexuales, cyborg o postgenéricos hacen uso de múltiples matices de la erótica, sexualidad y generización que ha supuesto la cibersociedad en la construcción del cuerpo-sexo-género en la Red» (García et al., 2004: 13).

Por otro lado, al contrario de leer en el manifiesto contra-sexual de Preciado una ansia de libertad y de transgresión de las identidades genéricas que oprimen los cuerpos y vidas de todos aquellos que no se ajustan a la norma, existen muchos/as autores/as que critican su propuesta. Hemos creído conveniente mencionar algunos aspectos de dicha crítica a través del artículo «Tengo, tengo, tengo... tú no tienes nada», o de los peligros de ciertas «prácticas subversivas»», de Garay (2004). En su artículo, dicho autor acusa a Preciado de intentar sustituir una norma por otra, critica la propuesta de Preciado, arguyendo que pretende sustituir una ley, esto es heterocentrada, por otra, la post-humana: «en la construcción misma de tal manifiesto se halla implícita la ineficaz estrategia de sustituir una naturaleza por otra-igualmente construida-, que deja las cosas tal cual están, sólo que en otras manos» (Garay, 2004: 188).

Como se desprende de la anterior afirmación, Garay considera que el texto de Preciado está pensado más como una prescripción para fundamentar una nueva hegemonía que sustituya a la heterocentrada, que para abrir paso a un nuevo mundo de posibilidades, libertades y goces. Así, subrayando el posible carácter prescriptivo de las teorías de Preciado, señala que lo único que se intenta con su manifiesto es cambiar el poder de manos, siendo esto una venganza de las minorías menospreciadas por el sistema heteronormativo:

La promulgación de hábitos que caracteriza a los principios de la sociedad contra-sexual (Preciado, 2002: 29-38) hace por fuerza desaparecer su capacidad perturbadora. El artículo 9 por ejemplo, en torno a la exigencia de control y regulación del tiempo, decreta que las actividades prescritas «(...) se practicarán regularmente un cierto número de horas al día (...)» (Preciado, 2002: 39). ¿Puede considerarse subversiva una práctica que exige la repetición sistemática de actividades regladas, entendidas como «trabajo social», «derecho y obligación» para cualquier cuerpo - «sujeto parlante»-? Cualquier alternativa deja de serlo en el momento en el que se convierte en la norma, en la nueva ley (Garay, 2004: 188).

Resulta interesante pues, traer a colación esta crítica a la propuesta contra-sexual de Preciado, ya que es una buena muestra de que su trabajo no deja impasible a nadie. Sin embargo, desde nuestra posición, entendemos que dichas propuestas no solamente resultan liberadoras para las minorías gays, lesbianas y transexuales; sino que abren un debate filosófico transgresor que enriquece la teoría feminista y la filosofía contemporánea, desmitificando la naturaleza de los cuerpos. En definitiva, sacando a relucir la artificialidad de nuestro sexo, nuestro cuerpo y nuestro género. ¿̨ué puede haber más transgresor que eso? 


\section{Conclusión}

A lo largo del presente escrito nos planteábamos como objetivo una aproximación a las ideas principales de la obra de Preciado, haciendo hincapié en el carácter transgresor de su propuesta. Como ya se ha tratado, para ir contra lo establecido, Preciado propone la formación de una nueva sociedad, la contra-sexual, y nos brinda las herramientas necesarias para caminar hacia su formación.

A través del concepto de prótesis de género, del dildo y del sexo como tecnología, Preciado desvela la artificialidad de los sexos, los géneros y los cuerpos, complejizando el eterno debate esencialismo versus constructivismo que ha protagonizado la teoría feminista desde hace décadas. En este sentido, «las prótesis, los complementos materiales o cualquier artículo resexualizado serán las herramientas básicas y fundamentales en la postpornografía y en la desgenitalización de las sexualidades. Serán denominadas, en palabras de Beatriz Preciado, como tecnologías del sexo (...)» (Flores Navarro, 2012: 81). Hemos visto pues, que el dildo permite desvelar la plasticidad de los sexos, destierra las antiguas nociones de placer y su consecuente parcelación y fijación en los órganos reproductores masculino y femenino:

El dildo juega con los roles de género, descentra el placer genital, y sustituye la concentración y producción de placer en un elemento extraño al cuerpo, un objeto protésico y posthumano. Al mismo tiempo desbanca la exclusividad de relaciones heterosexuales; provoca el fin de la diferencia sexual, de las relaciones de poder, y crea nuevas reglas de juego. En definitiva su relevancia reside en la capacidad de deconstrucción y resignificación de lo «marginal» (Cano, 2003: 151).

Así pues, si bien es cierto que las reflexiones filosóficas que hemos ido tratando a lo largo del texto socaban las bases del pensamiento occidental moderno y suponen una renovación de la agenda feminista actual, también lo es que su repercusión ha ido más allá del ámbito de pensamiento académico. Los temas tratados eran fruto de una necesidad, de dar respuesta a las reclamaciones realizadas por grupos minoritarios como los transexuales, transgénero, bollo, etc. En este sentido, el pensamiento de Preciado se ha convertido en la piedra angular de los movimientos queer, y ha permitido la eclosión de nuevos espacios de lucha en la calle, los centros culturales, los museos... al tiempo que ha ampliado los temas de interés en la academia, como por ejemplo, la pornografía. Se lucha pues contra la estigmatización de aquellas prácticas sexuales que se salgan de la norma, que son utilizadas como penalizaciones que a su vez refuerzan lo normativo. 
El sexo definido como tecnología; el reconocimiento político y social del transgenerismo y la transexualidad, a través de las luchas que dichos colectivos están protagonizando, como opciones políticas e identitarias; y la clara posibilidad de modificar los organismos humanos proporcionada por los avances tecnológicos quirúrgicos y hormonales, por nombrar algunos hechos, convierte cualquier afirmación sobre la existencia natural de dos únicos sexos en algo irrisorio y absurdo (Flores Navarro, 2012: 42).

Por último, nos gustaría terminar con unas palabras de Preciado en su obra Testo yonqui (2008), que revelan la esencia misma de su pensamiento, pero sobre todo de su lucha política canalizada a través de los movimientos queer, y ésta es desvelar que «no hay dos sexos, sino una multiplicidad de configuraciones genéticas, hormonales, cromosómicas, genitales, sexuales y sensuales. No hay verdad del género, de lo masculino y de lo femenino, fuera de un conjunto de ficciones culturales normativas» (Preciado, 2008: 175). Al final, todo es ficción.

\section{Bibliografía}

AgullerA, Rafael E. (2010): «Biopolítica, poder y sujeto en Michel Foucault», Universitas, Revista de Filosofía, Derecho y Política, N11 1, pp. 27-42.

BALZA, Isabel (201 1): «Ética corporal y sexuación: plasticidad y fluidez en el sujeto del posffeminismo», Revista Estudos Feministas, Vol.19 №1, pp. 21-33.

BUTLER, Judith (2002): Bodies that matter. On the discursive limits of sex, New York, Routledege.

CANO, Pilar (2003): «El dildo: un elemento queer en la historia del arte», MATERIA, No3, pp. 149-156.

CURIA, Dolores (2015): «La importancia de llamarse Paul. Entrevista con Paul B. Preciado», Página

12. Disponible en: http://www.pagina12.com.ar/diario/suplementos/soy/1-4022-201506-06.html

DUQUE, Carlos (2010): «Judith Butler y la teoría de la performatividad de género», Revista de Educación y Pensamiento, №17, pp. 85-95.

FERNÁNDEZ AGIS, Domingo (2009): «วQués es la biopolítica?», Cuadernos del Ateneo, No26, pp. 93-98.

FloRes NaVARRO, Helena (2012). La desobediencia sexual en el arte postpornográfico. Lucha polítia feminista contra la heteronormatividad patriarcal, Universidad de Granada / Lodz University.

Foucault, Michell (1977): Historia de la sexualidad Vol.I, México, Siglo XXI Editores.

GARAY, J. Alejandro (2004): «Tengo, tengo, tengo... tú no tienes nada», o de los peligros de ciertas «prácticas subversivas»», Quaderns de Filologia. Estudis Literaris, N9, pp. 185-197. 
García, Almudena; Moreno, Pilar, y SANChez, Jesús (2004): «Las Nuevas Identidades de Género en el Marco del Siglo XXI: del cyborg a las indetidades queer», Revista de Antropología Experimental, N'4, pp. 1-15.

Gu, Rosalind y ScharfF, Christina (eds.) (2011): New Femininities. Postfeminism, Neoliberalism and Subjectivity, New Hampshire, Palgrave Macmillan.

GIL, Rosalind (2014): «Posffeminist sexual culture» en CARTER, Cynthia; SteINER, Linda; y McLAughun, Lisa (Eds.): The Routledge Companion to Media and Gender, London and New York, Routledge, pp. 589-599.

HaraWAY, DonNa (1991): «A Cyborg Manifesto. Science, technology and socialist-feminism in the late twentieth century», en BEL, David y Barbara M. KENNEDY (Eds.): The Cybercultures Reader, London and New York, Routledge, pp.291-324.

López PASCUAL, Juliana (2012): «Entre el planteo filosófico y la praxis política: la obra de Beatriz Preciado y las micropolíticas de género», Claseshistoria, pp. 1-19.

LUMBY, Catherine (2014): «Post-posffeminism», en CARTeR, Cynthia; Steiner, Linda; y MCLAughun, Lisa (Eds.): The Routledge Companion to Media and Gender. London and New York, Routledge, pp. 600-609.

MARTínEZ, Ariel (2012): «El falo descentrado. Judith Butler, Donna Haraway y Beatriz Preciado: perspectivas conceptuales en torno al cuerpo», IV Congreso Internacional de Investigación y Práctica Profesional en Psicología XIX Jornadas de Investigación VIII Encuentro de Investigadores en Psicología del MERCOSUR, Buenos Aires.

PreCIADO, Paul B. (2002): Manifiesto contra-sexual, Madrid, Editorial Opera Prima.

PreCIADO, Paul B. (2006): «Basura y género. Mear/cagar. Maculino/Femenino», ESETÉ, Nº, pp. 40-49.

Preciado, Paul B. (2008): Testo Yonqui, Madrid, Espasa Calpe.

PRECIADO, Paul B. (2009): «Terror anal: apuntes sobre los primeros días de la revolución sexual», en HOCQUENGHeM, Guy (Ed.): El deseo homosexual, Editorial Melusina, Madrid, pp. 135-174.

RodríGuez, María Pilar (2003): "Crítica lesbiana: lecturas de la narrativa española contemporánea», Feminismo/s, N¹, pp. 87-102.

VARGAS-PARDO, Camilo (2008): «El «cuerpo parlante» en Flores, de Mario Bellatín», Cuadernos de Literatura, Vol. 13 No24, pp. 122-131.

Recibido el 10 de enero de 2016

Aceptado el 20 de febrero de 2016

BIBLID [1 139-1219 (2016) 21: 5-21] 